\title{
Which Mathematical and Physiological Formulas are Describing Voice Pathology: An Overview
}

Pedersen $\mathbf{M}^{*}$, Jønsson A, Mahmood S and Alexius Agersted A

The Medical Center, Oestergade 18, 1. 1100 Copenhagen, Denmark

\begin{abstract}
This study focuses upon changes in quantifiable parameters of voice production comparing normal voices and patients with complaints of hoarseness for more than two weeks. Acoustical signals and high speed films were data sources for mathematical and physiological formulas statistics of the voices. The software "Glottis Analysis Tools" (Erlangen, Germany) includes acoustical measurements and data sources in Glottal Area Waveforms (GAW) and Phonovibrograms (PVG), based on high speed film data. High speed film data were captured with high speed camera and software from Wolf Ltd, Germany. Data with statistical significant difference between 12 healthy voices and 12 patients with complaints of hoarse voices in a prospective case/control study were presented. The commonly used acoustical and physiological parameters showed hardly any statistical difference between the normal persons and the persons with complaints of hoarseness for more than two weeks. This suggests that evidence on physiological and acoustical measures of voice pathology is insufficient. Focus should be upon newer methods and tissue function.
\end{abstract}

Keywords: Voice Pathology; Phonovibrograms; Acoustical signals

\section{Introduction}

The inclusion criteria for this study were complaints of hoarseness for more than 2 weeks. The goal was to provide quantifiable protocols for determining if a voice was pathological or not. The following references were presented to show how far this field of research is in evidence:

In a Cochrane review the purpose was to assess the effectiveness of surgery versus non-surgical interventions for vocal cord nodules also diagnosed with acoustical measures and physiological voice diagnostics. No suitable trials were identified. No studies fulfilled the inclusion criteria of hoarseness and vocal nodules. It was concluded that there is a need for high-quality randomized controlled trials to evaluate the effectiveness of surgical and non-surgical treatment of vocal cord nodules [1].

Another study determined the reliability of objective voice measures of normal speaking voices used commonly in clinical practice of 18 healthy volunteers (nine males and nine females). Laryngeal efficiency and perturbation measures of fundamental frequency (F0) for both genders were made. For female cepstral peak prominence (CPP) had moderate reliability, whereas for males, the smooth CPP was reliable. A noise-to-harmonic ratio (NHRs) has the lowest consistency of all measures over the course. The authors concluded that additional research are needed to investigate which factors within the testing protocol and/or changes to the measurement instruments may lead to more consistent test results [2].

In a review focus was on evidence-based clinical voice assessment. The goal of the study was to determine what exists of research evidence, and to support the use of voice measures in the clinical assessment of patients with voice disorders. The literature provides some evidence for selected acoustic, laryngeal imaging-based, auditory-perceptual, functional, and aerodynamic measures to be used as components in a clinical based voice evaluation. The authors found a pressing need for high-quality research that is specially designed to expand the evidence base for clinical voice assessment [3].

Therefore, we made a comparison of normal persons versus patients with complaints of hoarseness in order to evaluate the possible validity of acoustical and video-derived physiological measurements in a prospective case control study, as a basis for more evidence related approaches of voice pathology.

\section{Material}

The prospective cohort study included 12 normal persons without voice complaint and 12 with hoarseness for more than 2 weeks. High speed films were made with the Wolf Ltd equipment and the "Glottis Analysis Tools" program were carried out on all 24 clients (Table 1) based on the combined hard/software. All 24 clients had data sets of 345 parameters, presenting our statistical material.

\section{Methods}

In the study mathematical and physiological formulas were focused upon from the high quality high speed films with 4000 pictures per second (Wolf Ltd. Germany) with the advanced software "Glottis Analysis Tools" (Erlangen Germany), including acoustical measurements and the following physiological data sources: Glottal Area Waveform (Figure 1), Trajectory-50\% (Figure 2) and Phonovibrograms (Figure 3). Attached to the scope is the microphone acquiring the acoustical signal (Wolf Ltd., Germany). In Table 1, an overview of the quantitative parameters is given. Due to the importance of the lack of evidence in acoustical formulas, we discussed some formulas in this study. Formulas and data sources were therefore presented. Many of the formulas were on different data sources (Glottal area waveform, trajectories $50 \%$ or acoustical measures) as they all are close to sinusoidal signals - that can be analyzed. This includes jitter and shimmer.

\section{Trajectories}

The image is an electronic representation of the rima glottidis. The dark

${ }^{*}$ Corresponding author: Pedersen M, MD. M. Eeg Cand. Stat, The Medica Center, Oestergade 18, 1. 1100 Copenhagen, Denmark, Tel: +4531126184; E-mail: m.f.pedersen@dadlnet.dk

Received April 19, 2016; Accepted May 20, 2016; Published May 27, 2016

Citation: Pedersen M, Jønsson A, Mahmood S, Agersted A A (2016) Which Mathematical and Physiological Formulas are Describing Voice Pathology: An Overview. J Gen Pract 4: 253. doi: 10.4172/2329-9126.1000253

Copyright: (c) 2016 Pedersen M, et al. This is an open-access article distributed under the terms of the Creative Commons Attribution License, which permits unrestricted use, distribution, and reproduction in any medium, provided the original author and source are credited. 
Citation: Pedersen M, Jønsson A, Mahmood S, Agersted AA (2016) Which Mathematical and Physiological Formulas are Describing Voice Pathology: An Overview. J Gen Pract 4: 253. doi: 10.4172/2329-9126.1000253

Page 2 of 6

\begin{tabular}{|c|c|c|c|}
\hline Source: Audio & Amplitude-Symmetry* & PPQ-11(\%) & Cycle-duration(ms) \\
\hline APF (\%) & Amplitude-Symmetry-Index & PPQ-3(\%) & EPF (\%) \\
\hline APQ-11(\%) & APF $(\%)$ & PPQ-5(\%) & EPQ-11(\%) \\
\hline APQ-3(\%) & APQ-11(\%) & PVI & EPQ-3(\%) \\
\hline APQ-5(\%) & APQ-3(\%) & RAP-v1 & EPQ-5(\%) \\
\hline AVI & APQ-5(\%) & RAP-v2 & Fundamental-Freq $(\mathrm{Hz})$ \\
\hline CHNR-v1(dB) & Asymmetrie-Quotient & Rate-Quotient(RQ) & Glottal-Area-Index(AC/OQ) \\
\hline CHNR-v2(dB) & AVI & Shim(\%) & Glottis-Gap-Index(GGI) \\
\hline $\mathrm{CPP}(\mathrm{dB})$ & CHNR-v1(dB) & SNR-v1(dB) & GNE \\
\hline Cycle-duration(ms) & CHNR-v2(dB) & SNR-v2(dB) & Harmonics-Intensity(\%) \\
\hline $\operatorname{EPF}(\%)$ & Closing-Quotient(CIQ) & Spatial-Symmetry* & $\mathrm{HNR}(\mathrm{dB})$ \\
\hline EPQ-11(\%) & $\mathrm{CPP}(\mathrm{dB})$ & Spatial-Symmetry-Index & Jitt(\%) \\
\hline EPQ-3(\%) & Cycle-duration(ms) & Spectral-Flatness(SFM) & Jitt-Factor \\
\hline EPQ-5(\%) & DynamicRange-Symmetry* & Speed-Index(SI) & Jitt-Ratio \\
\hline Fundamental-Freq(Hz) & DynamicRange-Symmetry-Index & Speed-Quotient(SQ) & max-Harmonic $(\mathrm{Hz})$ \\
\hline GNE & $\operatorname{EPF}(\%)$ & Stiffness & Maximum-Area-Declination-Rate \\
\hline Harmonics-Intensity(\%) & $E P Q-11(\%)$ & Time-Periodicity & max-WMC \\
\hline $\mathrm{HNR}(\mathrm{dB})$ & $E P Q-3(\%)$ & Waveform-Symmetry-Index & mean-Jitt(ms) \\
\hline Jitt(\%) & $E P Q-5(\%)$ & Souce: Phonovibrogram (PVG) & mean-Shim $(\mathrm{dB})$ \\
\hline Jitt-Factor & Fundamental-Freq(Hz) & ContourAngles-Symmetry* & mean-WMC \\
\hline Jitt-Ratio & Glottal-Area-Index(AC/OQ) & ContourAngles-Symmetry-Index & min-Subharmonic(Hz) \\
\hline max-Harmonic(Hz) & Glottis-Gap-Index(GGI) & Contour-Angle(DEG) & $\mathrm{NNE}(\mathrm{dB})$ \\
\hline max-WMC & GNE & Source: Trajectories $\mathbf{5 0 \%}$ & Open-Quotient(OQ) \\
\hline mean-Jitt(ms) & Harmonics-Intensity (\%) & Amplitude-Symmetry* & Peak-Acceleration \\
\hline mean-Shim(dB) & $\mathrm{HNR}(\mathrm{dB})$ & Amplitude-Symmetry-Index & Peak-Closing-Velocity \\
\hline mean-WMC & Jitt (\%) & DynamicRange-Symmetry* & Plateau-Quotient(PQ) \\
\hline min-Subharmonic $(\mathrm{Hz})$ & Jitt-Factor & DynamicRange-Symmetry-Index & PPF (\%) \\
\hline $\mathrm{NNE}(\mathrm{dB})$ & Jitt-Ratio & Phase-Asymmetry* & PPQ-11(\%) \\
\hline $\operatorname{PPF}(\%)$ & max-Harmonic(Hz) & Phase-Asymmetry-Index & PPQ-3(\%) \\
\hline PPQ-11(\%) & Maximum-Area-Declination-Rate & Waveform-Symmetry-Index & PPQ-5(\%) \\
\hline PPQ-3(\%) & $\max -\mathrm{WMC}$ & Amplitude-Length-Ratio & PVI \\
\hline PPQ-5(\%) & mean-Jitt(ms) & Amplitude-Periodicity & RAP-v1 \\
\hline $\mathrm{PVI}$ & mean-Shim(dB) & Amplitude-Quotient & RAP-v2 \\
\hline RAP-v1 & mean-WMC & APF $(\%)$ & Rate-Quotient(RQ) \\
\hline RAP-v2 & min-Subharmonic(Hz) & APQ-11(\%) & Shim (\%) \\
\hline Shim (\%) & $\mathrm{NNE}(\mathrm{dB})$ & APQ-3(\%) & SNR-v1(dB) \\
\hline SNR-v1(dB) & Open-Quotient(OQ) & APQ-5(\%) & SNR-v2(dB) \\
\hline SNR-v2(dB) & Peak-Acceleration & Asymmetrie-Quotient & Spectral-Flatness(SFM) \\
\hline Spectral-Flatness(SFM) & Peak-Closing-Velocity & AVI & Speed-Index(SI) \\
\hline Source: GAW & Phase-Asymmetry* & CHNR-v1(dB) & Speed-Quotient(SQ) \\
\hline Amplitude-Length-Ratio & Phase-Asymmetry-Index & CHNR-v2(dB) & Stiffness \\
\hline Amplitude-Periodicity & Plateau-Quotient(PQ) & Closing-Quotient (CIQ) & Time-Periodicity \\
\hline Amplitude-Quotient & PPF (\%) & $\mathrm{CPP}(\mathrm{dB})$ & \\
\hline
\end{tabular}

Table 1: Overview of some measured parameters in "Glottal Analysis Tools" used for 12 normal persons compared with 12 patients with complaints of hoarseness for more than two weeks $[4,5]$

blue line defines the left vocal fold. The red line delimits the right vocal fold. The blue dotted line in the middle is the center line between the vocal folds. The vocal fold movements are calculated from this line.

The left chart illustrates a computed cycle. The dark blue curve is the left vocal fold fluctuation, and the red curve is the right vocal fold fluctuation.

$50 \%$ is an indication that the chart depicts the vocal folds in $50 \%$ distance from the posterior limit (and therefore 50\% distance to the anterior limit) $=$ trajectory- $50 \%$.

The purple line in the computed image indicates, where Traj-50\% downloads the numbers from.
Examples and formulas from Table 1 are given:

\section{Cepstral harmonics-to-noise ratio (CHNR)}

$$
C H N R-v 2(d B)=10 \cdot \log _{10}\left[\frac{\sum_{n=1}^{H_{\max }}\left|\vec{H}\left(n \cdot{ }_{0}\right)\right|^{2}}{\left.\left.a \sum_{n=1}^{H_{\max }} 10^{\left(2 \cdot B_{L}(n \log )-F\right.} n_{n_{0}+}\left(\cdot{ }_{0}\right)\right|^{2}\right)}\right]
$$

\section{Cepstral peak prominence}

CPP $(d B)$ is defined as the difference in amplitude between the cepstral peak and the corresponding value on the regression line computed between $1 \mathrm{~ms}$ and the maximum quefrency (i.e., the predicted cepstral magnitude for the quefrency at the cepstral peak) [4]. 

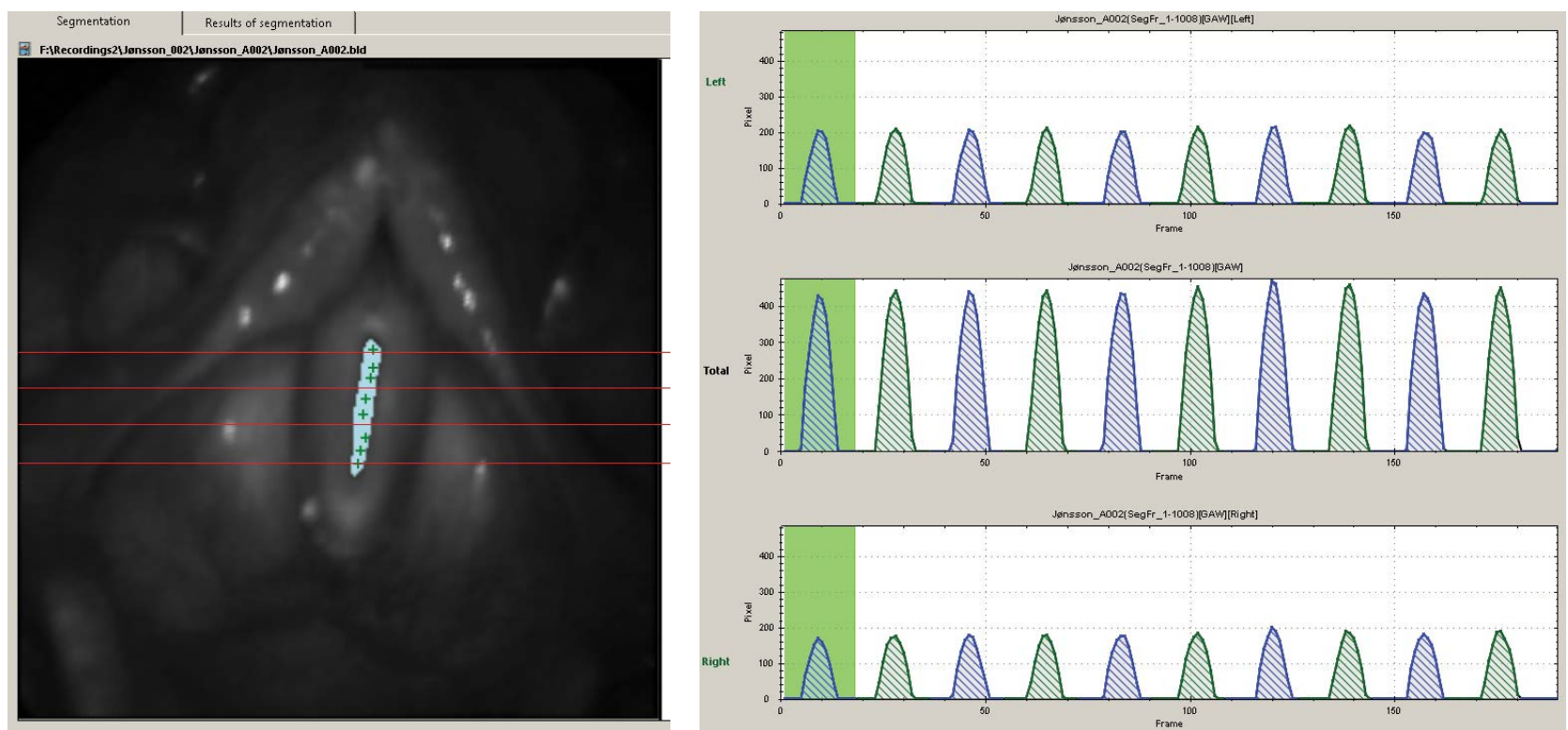

Figure 1: Glottal Area Waveform presentation. "Space curves" - the area between the vocal folds is calculated and plotted in a curve. The curves switch between green and blue to indicate different cycles of vocal fold movement in the software system from Erlangen, Germany.

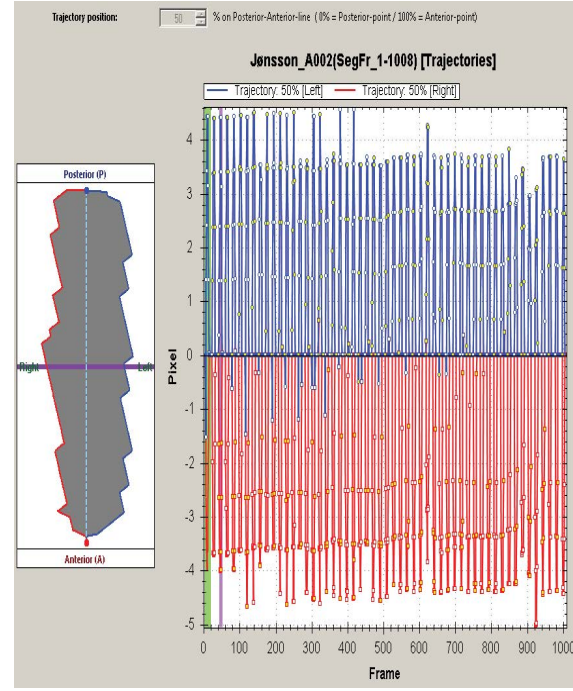

Figure 2: Trajectories ("Quantitative kymography").

\section{Contour angles of phonovibrograms (PVG)}

Contour-Angles (deg) are calculated in both anterior and posterior parts during opening as well as closing of vocal folds for the left and right side of PVG, respectively. Hence, $C A_{i}^{\text {side, Item }}$ denotes the Contour-Angles for $i^{\text {th }}$ cycle, where side represents the corresponding side of PVG: $L$ for Left side and $R$ for Right side. Item signifies the position of related ContourAngle: $O A$ : Opening - Anterior, OP: Opening - Posterior CA: Closing Anterior and $O P$ : Closing - Posterior

\section{Energy perturbation quotient $5 \%$ \& $11 \%$}

$$
E P Q(\%)=\frac{1}{N-k} \sum_{i=\frac{k-1}{2}}^{N-\frac{k-1}{2}-1}\left|1-\frac{k \cdot E(i)}{\sum_{j=-\frac{k-1}{2}}^{\frac{k-1}{2}} E(i+j)}\right| \cdot 100
$$

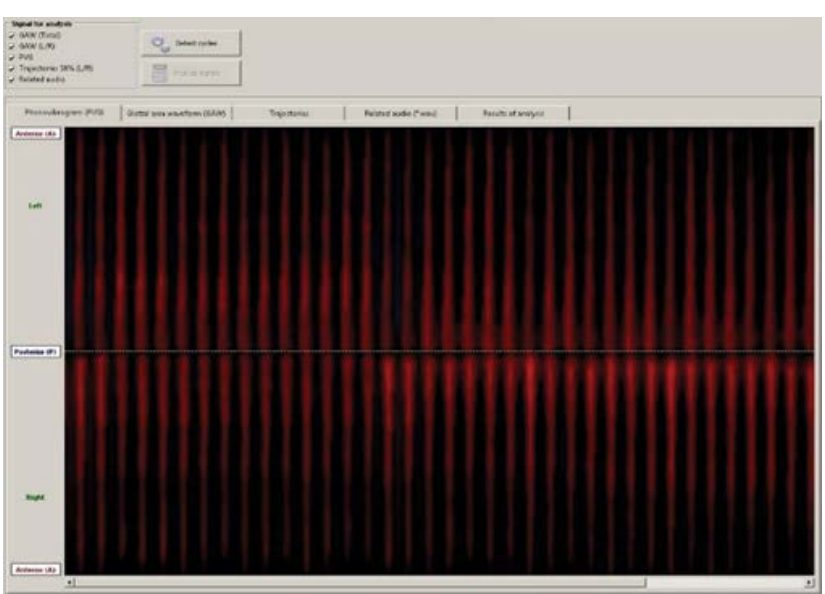

Figure 3: High speed films with phonovibrogram of single movements of the right and left vocal folds. Phonovibrogram of a contest winning female, showing the regularity of single movement of the right and left vocal folds.

Where $k$ represents the number of cycles considered for computation of quotients: $k=3$ : EPQ-3 (\%), $k=5$ : EPQ-5 (\%) and $k=11$ : EPQ-11 (\%). Furthermore, $E(i)$ - signal energy within a $i^{\text {th }}$ cycle and $N$ - the number of analyzed cycles (equivalent to the number of elements $E$ ).

In Glottis Analysis Tools the following energy-related parameters are calculated:

\section{Harmonics intensity}

$$
\text { Harmonics - Intensity }(\%)=100 \cdot \frac{\sum_{n=2}^{H m a x} \mid \boldsymbol{F}\left(n \cdot{ }_{0} \mid\right.}{\sum_{i \geq 1}|\boldsymbol{H}(\quad)|}
$$

These measures can be calculated for the following signals: Glottal area waveform (GAW), Acoustics and Glottal trajectories. 
Citation: Pedersen M, Jønsson A, Mahmood S, Agersted AA (2016) Which Mathematical and Physiological Formulas are Describing Voice Pathology: An Overview. J Gen Pract 4: 253. doi: 10.4172/2329-9126.1000253

Page 4 of 6

\begin{tabular}{|c|c|c|c|c|c|c|c|c|}
\hline & Parameter & Source & Type & $\begin{array}{l}\text { Mean difference } \\
\text { healthy-hoarse }\end{array}$ & Standard Error & DF & T Value & $\operatorname{Pr}>|T|$ \\
\hline 1 & $\begin{array}{l}\text { Cepstral Harmonics-to- } \\
\text { Noise Ratio-v2 (dB) }\end{array}$ & [GAW] & & 10,63 & 4,41 & 22 & 2,41 & 0,02 \\
\hline 2 & $\begin{array}{l}\text { Cepstral Harmonics-to- } \\
\text { Noise Ratio-v2 (dB) }\end{array}$ & [GAW] & [Left] & 11,89 & 4,81 & 20 & 2,47 & 0,02 \\
\hline 3 & $\begin{array}{l}\text { Cepstral Harmonics-to- } \\
\text { Noise Ratio-v2(dB) }\end{array}$ & [GAW] & [Right] & 8,56 & 4,21 & 22 & 2,03 & 0,05 \\
\hline 4 & $\begin{array}{l}\text { Cepstral Harmonics-to- } \\
\text { Noise Ratio-v2 (dB) }\end{array}$ & [Traj-50\%] & [Left] & 10 & 4,33 & 21 & 2,31 & 0,03 \\
\hline 5 & $\begin{array}{l}\text { Cepstral Peak } \\
\text { Prominence }(\mathrm{dB})\end{array}$ & [GAW] & [Left] & 0,58 & 0,26 & 20 & 2,2 & 0,04 \\
\hline 6 & $\begin{array}{l}\text { Cepstral Peak } \\
\text { Prominence }(\mathrm{dB})\end{array}$ & [Traj-50\%] & [Right] & 0,33 & 0,17 & 22 & 2 & 0,06 \\
\hline 7 & Contour-Angle(DEG) & {$[P \vee G]$} & [Left] & 10,23 & 4,3 & 20 & 2,38 & 0,03 \\
\hline 8 & $\begin{array}{l}\text { Energy Perturbation } \\
\text { Quotient-5 (\%) }\end{array}$ & [Traj-50\%] & [Left] & $-9,06$ & 3,53 & 21 & $-2,56$ & 0,02 \\
\hline 9 & Harmonics-Intensity (\%) & [GAW] & & 4,1 & 1,45 & 22 & 2,83 & 0,01 \\
\hline 10 & Harmonics-Intensity (\%) & [GAW] & [Left] & 3,17 & 1,25 & 20 & 2,53 & 0,02 \\
\hline 11 & Harmonics-Intensity (\%) & [GAW] & [Right] & 3,41 & 1,41 & 22 & 2,42 & 0,02 \\
\hline 12 & Harmonics-Intensity (\%) & [Traj-50\%] & [Left] & 2,8 & 1,3 & 21 & 2,16 & 0,04 \\
\hline 13 & $\begin{array}{l}\text { Normalized Noise Energy } \\
\text { (dB) }\end{array}$ & [GAW] & [Left] & $-3,38$ & 1,39 & 20 & $-2,42$ & 0,03 \\
\hline 14 & $\begin{array}{l}\text { Period Perturbation } \\
\text { Quotient-11(\%) }\end{array}$ & [GAW] & [Left] & $-1,89$ & 0,84 & 19 & $-2,25$ & 0,04 \\
\hline 15 & $\begin{array}{l}\text { Period Perturbation } \\
\text { Quotient-11(\%) }\end{array}$ & [GAW] & [Right] & $-2,17$ & 0,93 & 21 & $-2,33$ & 0,03 \\
\hline 16 & $\begin{array}{l}\text { Signal-to-Noise Ratio- } \\
\text { v1(dB) }\end{array}$ & [GAW] & & 1,15 & 0,56 & 22 & 2,06 & 0,05 \\
\hline 17 & $\begin{array}{l}\text { Signal-to-Noise Ratio- } \\
\text { v1(dB) }\end{array}$ & [GAW] & [Left] & 1,32 & 0,6 & 20 & 2,19 & 0,04 \\
\hline 18 & $\begin{array}{l}\text { Signal-to-Noise Ratio- } \\
\mathrm{v} 1(\mathrm{~dB})\end{array}$ & [GAW] & [Right] & 1,03 & 0,51 & 22 & 2,01 & 0,06 \\
\hline 19 & Spectral-Flatness(SFM) & [GAW] & & $-2,74$ & 1,2 & 22 & $-2,28$ & 0,03 \\
\hline 20 & $\begin{array}{l}\text { minimum- } \\
\text { Subharmonic(Hz) }\end{array}$ & [GAW] & & $-81,06$ & 40,25 & 22 & $-2,01$ & 0,06 \\
\hline 21 & $\begin{array}{l}\text { minimum- } \\
\text { Subharmonic }(\mathrm{Hz})\end{array}$ & [GAW] & [Right] & $-83,42$ & 39,61 & 22 & $-2,11$ & 0,05 \\
\hline 22 & $\begin{array}{l}\text { minimum- } \\
\text { Subharmonic(Hz) }\end{array}$ & [Traj-50\%] & [Left] & $-153,85$ & 23,88 & 21 & $-6,44$ & $<, 0001$ \\
\hline
\end{tabular}

Table 2: "Glottis Analysis Tools" measures analyze in an analysis of variance estimating mean difference between healthy and hoarse persons (adjusting for gender), in a prospective case control study of 12 normal persons and 12 patients with complaints of hoarseness for more than two weeks.

Furthermore: $F(k)-k^{\text {th }}$ coefficient of Fourier transform of the signal $\left(\mathrm{k}=0\right.$ - the DC component) and $C(k)-k^{\text {th }}$ Cepstrum coefficient $\overline{\mathbb{L}}()=\mathcal{F}^{-1}\left\{10 \cdot \log _{15} f\left(|\grave{u}()|^{2}\right)\right\}$

$\omega_{0}$ - index of Fourier coefficient represents fundamental frequency $\left(f_{0}\right), H \max$ - maximum order of harmonics for $f_{0}, \omega_{\min }-$ index $f f$ Fourier coefficient represents minimum occurring subharmonic for $f_{0}$.

\section{Normalized noise energy}

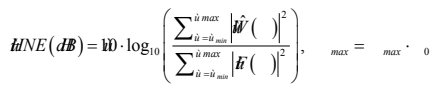

\section{Signal-to-noise ratio}

$$
S N R-v 1(d B)=10 \cdot \log _{10}\left(\frac{E_{t}}{E_{t}-E_{h}}\right)
$$

$$
S N R-v 2(d B)=20 \cdot \log _{10}\left[\frac{\sqrt{\sum_{t=1}^{N}|f(t)|^{2}}}{\sqrt{\sum_{t=1}^{N}|n(t)|^{2}}}-1\right]
$$

\section{Spectral flatness}

$$
\text { Epectral - Flatness }(S F M) \neq i \frac{20}{N} \cdot\left(\sum_{i=1}^{\frac{N}{2}} \log _{10}|F()|^{2}\right)-10 \cdot \log _{10}\left(\frac{2}{N} \cdot \sum_{i=1}^{N / 2}|()|^{2}\right)
$$

\section{Minimum subharmonics}

Min-Subharmonic $(\mathrm{Hz})$ - minimum occurring subharmonic frequency (fundamental frequency is the multiple of this frequency) in Hz. Further formulas are presented: Jitter \% because it is commonly used, Shimmer \% because it is commonly used, Stiffness because it might be interesting in singers and Amplitude symmetry index because earlier analyses showed signs of significance.

\section{Jitter \%}

$$
\operatorname{Jitter}(\%)=\frac{\text { Mean Jitter }(m s)}{\frac{1}{N} \sum_{i=0}^{N-1} p(i)} \cdot 100
$$

Shimmer, mean \& shimmer\%

$$
\begin{aligned}
& \text { Mean-Shimmer }(d B)=\frac{20}{N-1} \sum_{i=0}^{N-2}\left|\frac{A(i)}{A(i+1)}\right| \\
& \text { Shimmer }(\%)=\frac{\text { Mean-shimmer }(d B)}{\frac{20}{N} \sum_{i=0}^{N-1} \log _{10} A(i)} \cdot 100
\end{aligned}
$$


Shimmer is strength variation and it is measured at the maximum amplitude of all measuring points. A(i) is the dynamic range (max$\mathrm{min}$ ) of the $\mathrm{i}^{\text {th }}$ cycle and $\mathrm{N}$ is the number of analyzed cycles (equivalent to the number of elements in $\mathrm{A}(\mathrm{i})$ ).

Stiffness (from data sources Glottal Area Waveform (GAW) and traj-50\%)

$$
\text { Stiffness }=\frac{\max _{t \in T_{i}}(s(t))}{A_{i}}
$$

Where $\mathrm{T}_{\mathrm{i}}$ is the duration of the $\mathrm{i}^{\text {th }}$ cycle in milliseconds $(\mathrm{ms}) . \mathrm{A}^{\mathrm{i}}$ is the dynamic range ( $\max -\min$ ) of $\mathrm{i}^{\text {th }} \mathrm{cycle} . \mathrm{s}(\mathrm{t})$ is the magnitude of the $1^{\text {st }}$ derivative of the considered signal for $i^{\text {th }}$ cycle $\left(t \subset T_{i}\right)$.

\section{Amplitude symmetry index (GAW and traj-50\%)}

$$
\text { Amplitude Symmetry Index }=\frac{\min \left(\max \left[G A_{i}^{L}\right], \max \left[G A_{i}^{R t}\right]\right)}{\max \left(\max \left[G A_{i}^{L}\right], \max \left[G A_{i}^{R}\right]\right.}
$$

$\mathrm{GA}_{\mathrm{i}}=$ Glottal area waveform for the $\mathrm{i}^{\text {th }}$ cycle, $\mathrm{L}=$ Left side and $\mathrm{R}=$ Right side.

\section{Results}

Results of the calculation with Glottis Analysis Tools were made on 12 healthy voices, and 12 patients with complaints of hoarseness for more than two weeks in a prospective case/control study of the given parameters (Table 1). Spearman correlation between variables related to the high speed films and acoustic measurements made at the same was calculated for a total of 345 combinations. The variables related to the high speed films were analyzed in an analysis of variance including gender and hoarse/healthy as fixed effects. As a measure of diagnostic value, the mean difference between the population of hoarse and population of healthy persons have been estimated and is shown in (Table 2) for the variables with the most statistical difference. Similarly, Table 3a shows the mean difference between hoarse and healthy persons for the commonly used parameters of Jitter and Shimmer. Table $3 \mathrm{~b}$ is a continuation of commonly used parameters - between 12 normal persons and 12 persons with hoarseness. Figure 4 shows a scatterplot of parameter with the most statistical difference between hoarse and healthy persons.

The first purpose was to characterize the distribution of the parameters, not to compare the two groups. To our knowledge, the study will be the first of its nature to describe the parameters and therefore the study will provide important contribution to generate hypothesis in future research which include a bigger amount of persons to show the differences of voice pathology. There was no significant difference between males and females.

\section{Discussion and Conclusion}

The "Glottis Analysis Tools" analysis program is one of the most updated voice analysis program and an interesting supplement of acoustical and physiological voice analysis, as it operates on vocal fold level in comparison with acoustical analysis on high speed films. The prognostic values of the results are important. Jitter and shimmer and many other acoustical measurements have been shown not to differentiate between healthy and hoarse persons. A few of the comparisons between hoarse and healthy persons have some significance (Table 2). Maybe they can be used to compare difference objective measurements measures in the future [5]. Till now estimates of levels of hoarseness are not optimal. The acoustical measures of voices show very little statistical differences between 12 normal persons and 12 patients with complaints of hoarseness in our prospective case

\begin{tabular}{|c|c|c|c|c|c|c|c|}
\hline Parameter & Source & Type & Estimate & Standard Error & DF & t Value & $\operatorname{Pr}>|t|$ \\
\hline Jitt(\%) & [Audio] & & 0,31 & 3,56 & 22 & 0,09 & 0,93 \\
\hline Jitt(\%) & [GAW] & & $-1,42$ & 1,44 & 22 & $-0,99$ & 0,33 \\
\hline Jitt(\%) & [GAW] & [Left] & $-1,84$ & 1,51 & 20 & $-1,23$ & 0,23 \\
\hline Jitt(\%) & [GAW] & [Right] & $-2,04$ & 1,32 & 22 & $-1,55$ & 0,14 \\
\hline Jitt(\%) & [Traj-50\%] & [Left] & $-0,74$ & 1,87 & 21 & $-0,39$ & 0,7 \\
\hline Jitt(\%) & [Traj-50\%] & [Right] & $-1,32$ & 1,46 & 22 & $-0,9$ & 0,38 \\
\hline Jitt-Factor & [Audio] & & 0,44 & 3,61 & 22 & 0,12 & 0,9 \\
\hline Jitt-Factor & [GAW] & & $-1,6$ & 1,47 & 22 & $-1,09$ & 0,29 \\
\hline Jitt-Factor & [GAW] & [Left] & $-2,03$ & 1,54 & 20 & $-1,32$ & 0,2 \\
\hline Jitt-Factor & [GAW] & [Right] & $-2,08$ & 1,29 & 22 & $-1,62$ & 0,12 \\
\hline Jitt-Factor & [Traj-50\%] & [Left] & $-0,65$ & 1,9 & 21 & $-0,34$ & 0,74 \\
\hline Jitt-Factor & [Traj-50\%] & [Right] & $-1,38$ & 1,5 & 22 & $-0,92$ & 0,37 \\
\hline Jitt-Ratio & [Audio] & & 3,1 & 35,61 & 22 & 0,09 & 0,93 \\
\hline Jitt-Ratio & [GAW] & & $-14,18$ & 14,38 & 22 & $-0,99$ & 0,34 \\
\hline Jitt-Ratio & [GAW] & [Left] & $-18,45$ & 15,06 & 20 & $-1,23$ & 0,23 \\
\hline Jitt-Ratio & [GAW] & [Right] & $-20,37$ & 13,17 & 22 & $-1,55$ & 0,14 \\
\hline Jitt-Ratio & [Traj-50\%] & [Left] & $-7,37$ & 18,67 & 21 & $-0,39$ & 0,7 \\
\hline Jitt-Ratio & [Traj-50\%] & [Right] & $-13,19$ & 14,59 & 22 & $-0,9$ & 0,38 \\
\hline Shim (\%) & [Audio] & & 1,27 & 21,82 & 22 & 0,06 & 0,95 \\
\hline Shim (\%) & [GAW] & & $-0,61$ & 0,54 & 22 & $-1,13$ & 0,27 \\
\hline Shim (\%) & [GAW] & [Left] & $-1,21$ & 0,65 & 20 & $-1,86$ & 0,08 \\
\hline Shim (\%) & [GAW] & [Right] & $-0,73$ & 0,86 & 22 & $-0,84$ & 0,41 \\
\hline Shim (\%) & [Traj-50\%] & [Left] & $-6,53$ & 6,34 & 21 & $-1,03$ & 0,31 \\
\hline Shim (\%) & [Traj-50\%] & [Right] & 1,9 & 4,07 & 22 & 0,47 & 0,64 \\
\hline
\end{tabular}
control study. This seems to further establish that voice measures

Table 3a: The commonly used parameters of Jitter and Shimmer shows no statistical difference in "Glottis Analysis Tools" between 12 normal persons and 12 persons with complaints of hoarseness in a prospective case control study (SAS program 9,4 F-test, adjusted for gender). 
Citation: Pedersen M, Jønsson A, Mahmood S, Agersted AA (2016) Which Mathematical and Physiological Formulas are Describing Voice Pathology: An Overview. J Gen Pract 4: 253. doi: 10.4172/2329-9126.1000253

Page 6 of 6

\begin{tabular}{|c|c|c|c|c|c|c|c|}
\hline Parameter & Source & Type & Estimate & Standard Error & DF & t Value & $\operatorname{Pr}>|t|$ \\
\hline Stiffness & [GAW] & & 0,01 & 0,02 & 20 & 0,57 & 0,57 \\
\hline Stiffness & [GAW] & [Left] & 0,02 & 0,03 & 18 & 0,58 & 0,57 \\
\hline Stiffness & [GAW] & [Right] & 0,01 & 0,03 & 20 & 0,37 & 0,72 \\
\hline Stiffness & [Traj-50\%] & [Left] & $-0,01$ & 0,03 & 19 & $-0,21$ & 0,84 \\
\hline Stiffness & [Traj-50\%] & [Right] & 0 & 0,03 & 20 & $-0,15$ & 0,88 \\
\hline Amplitude-Length-Ratio & [GAW] & & $-0,24$ & 0,55 & 20 & $-0,44$ & 0,66 \\
\hline Amplitude-Length-Ratio & [GAW] & [Left] & $-0,05$ & 0,32 & 18 & $-0,16$ & 0,87 \\
\hline Amplitude-Length-Ratio & [GAW] & [Right] & $-0,31$ & 0,33 & 20 & $-0,93$ & 0,36 \\
\hline Amplitude-Length-Ratio & [Traj-50\%] & [Left] & $-0,01$ & 0,01 & 19 & $-0,92$ & 0,37 \\
\hline Amplitude-Length-Ratio & [Traj-50\%] & [Right] & $-0,02$ & 0,01 & 20 & $-1,6$ & 0,12 \\
\hline Amplitude-Periodicity & [GAW] & & 0,03 & 0,03 & 20 & 1,16 & 0,26 \\
\hline Amplitude-Periodicity & [GAW] & [Left] & 0,05 & 0,03 & 18 & 1,82 & 0,09 \\
\hline Amplitude-Periodicity & [GAW] & [Right] & 0,03 & 0,03 & 20 & 0,98 & 0,34 \\
\hline Amplitude-Periodicity & [Traj-50\%] & [Left] & 0,03 & 0,03 & 19 & 1,19 & 0,25 \\
\hline Amplitude-Periodicity & [Traj-50\%] & [Right] & 0,02 & 0,03 & 20 & 0,48 & 0,63 \\
\hline Amplitude-Quotient & [GAW] & & 0,11 & 0,31 & 20 & 0,35 & 0,73 \\
\hline Amplitude-Quotient & [GAW] & [Left] & 0,01 & 0,32 & 18 & 0,05 & 0,96 \\
\hline Amplitude-Quotient & [GAW] & [Right] & 0,04 & 0,35 & 20 & 0,1 & 0,92 \\
\hline Amplitude-Quotient & [Traj-50\%] & [Left] & 0,01 & 0,26 & 19 & 0,05 & 0,96 \\
\hline Amplitude-Quotient & [Traj-50\%] & [Right] & $-0,2$ & 0,29 & 20 & $-0,7$ & 0,49 \\
\hline Amplitude-Symmetry* & [GAW] & & 0,1 & 0,13 & 20 & 0,76 & 0,46 \\
\hline Amplitude-Symmetry* & [Traj-50\%] & & $-1316,17$ & 1447,91 & 20 & $-0,91$ & 0,37 \\
\hline $\begin{array}{l}\text { Amplitude-Symmetry- } \\
\text { Index }\end{array}$ & [GAW] & & 0,03 & 0,04 & 20 & 0,79 & 0,44 \\
\hline $\begin{array}{l}\text { Amplitude-Symmetry- } \\
\text { Index }\end{array}$ & [Traj-50\%] & & 0,07 & 0,07 & 20 & 1,07 & 0,3 \\
\hline
\end{tabular}

Table 3b: Commonly used parameters continued (SAS program 9,4 F-test, adjusted for gender).

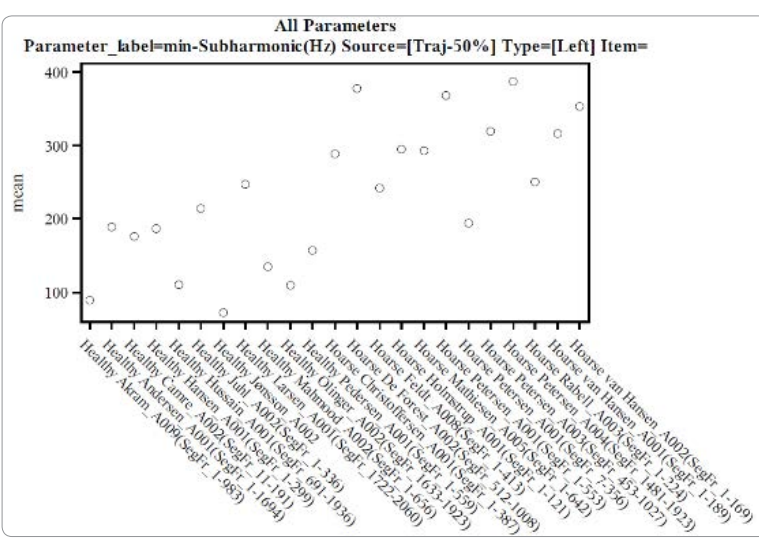

Figure 4: Minimum Subharmonics for left vocal fold presenting 12 normal persons and 12 persons complaining of hoarseness.

till now are not clinically evidence based as such. Some glottal area waveform measures are of interest but randomized studies are lacking. The new methods should be focused upon: Overtones/ harmonics [6] as well as tissue evaluation and Narrow Band Imaging [7], as well as Optical Coherence Tomography [8].

\section{References}

1. Pedersen M, Glashan J (2012) Surgical versus non-surgical interventions for vocal cord nodules (Review) The Cochrane Library 1-13.

2. Leong K, Hawkshaw M J, Dentchev D, Gupta R, Lurie D, et al. (2012) Reliability of objective voice measures of normal speaking voices, J Voice 27: 170-176.

3. Roy N, Barkmeier-Kraemer J, Eadie T, Sivasankar MP, Mehta D, et al. (2013) Evidence-based clinical voice assessment: a systematic review, Am J Speech
Lang Pathol 22: 212-226.

4. Glottis analysis tools. (2014) Universitätsklinikum Erlangen, Germany

5. Christopher B, Angelika K, Eysholdt U, Ziethe A, Döllinger M (2013) Quantitative analysis of organic vocal fold pathologies in females by high-speed endoscopy. The laryngoscope 123: 1686-1693.

6. Donald Gray Miller (2008) Resonance in singing. Voicevista. Inside View Press, and Sygyt Ltd

7. Ochsner MC, Klein AM (2015) The utility of narrow band imaging in the treatment of laryngeal papillomatosis in awake patients. Journal of voice 29 . 349-51. Brian J. F. Wong, Zhongping Chen, Usama Mahmood BS (2006) Optical Coherence Tomography of Laryngeal Cancer. The Laryngoscope 116 1107-1113. 